\title{
Pólya urn with memory kernel and asymptotic behaviors of autocorrelation function
}

\author{
Shintaro Mori* \\ Department of Mathematics and Physics, \\ Faculty of Science and Technology, Hirosaki University, \\ Bunkyo-cho 3, Hirosaki, Aomori 036-8561, Japan \\ Masato Hisakadd $\dagger$ \\ Nomura Holdings Inc., \\ Otemachi 2-2-2, Chiyoda-ku, \\ Tokyo 100-8130, Japan \\ Kazuaki Nakayamał \\ Department of Mathematical Sciences, \\ Faculty of Science, Shinshu University, \\ Asahi 3-1-1, Matsumoto, Nagano 390-8621, Japan
}

(Dated: September 6, 2021) 


\begin{abstract}
Pólya urn is a stochastic process in which balls are randomly drawn from an urn of red and blue balls, and balls of the same color as the drawn balls are added. The probability of a ball of a certain color being drawn is equal to the percentage of balls of that color in the urn. We introduce arbitrary memory kernels to modify this probability. If the memory kernel decays exponentially, it is a stationary process and is mean-reverting. If the memory kernel decays by a power-law, a phase transition occurs and the asymptotic behavior of the autocorrelation function changes. An auxiliary field variable is introduced to transform the process Markovian and the field obeys a multivariate Ornstein-Uhlenbeck process. The exponents of the power law are estimated for the decay of the leading and subleading terms of the autocorrelation function. It is shown that the power law exponents changes discontinuously at the critical point.

PACS numbers: $05.70 . \mathrm{Fh}, 89.65 . \mathrm{Gh}$
\end{abstract}

$*$ shintaro.mori@hirosaki-u.ac.jp
$\dagger$ hisakadom@yahoo.co.jp
$\ddagger$ nakayama@math.shinshu-u.ac.jp 


\section{INTRODUCTION}

Econophysics and socio-physics are fascinating research fields in statistical physics [1 $[3]$. In particular, herding or the tendency to follow the majority has attracted many researchers as it plays a crucial role in the understanding of the phenomena [4 7]. Many types of probabilistic models have been proposed to model herding and one example is the ant recruitment model, which describes the intermittent oscillation of the ants faced with two identical food sources [8]. It incorporates a simple herding mechanism where a randomly chosen ant chooses one of the two food sources with a probability that is proportional to the numbers of ants who have chosen the food sources.

A variant of the ant recruitment model has been adopted as a model for the intermittent switching phenomena of trading strategy, trend-follower and contrarian, in the modeling of the time-series data of stock and foreign exchange markets[9]. Another application is the collective bankruptcy of assets[10]. In the modeling of the time-series data of defaults of assets, the estimation of the default probability (PD) and the default correlation is crucial as it affects the risk estimation of the investment. In addition to the default correlation for the defaults in the same year, it is necessary to model the correlation of the defaults in different years. The beta-binomial distribution model with memory kernel is a model for both correlations and it is tractable compared to the established model based on the correlation of asset values [11, 12].

The fascinating point of the beta-binomial distribution model with memory kernel is its phase transition, and its essence is grasped by a variant of the Pólya urn[13], Pólya urn with memory kernel[10]. If the memory kernel decays with the power law, the power-law index $\gamma$ determines the phases of the model. If $\gamma>1$, the autocorrelation function (ACF) shows power-law decay with exponent $\delta=\gamma$. For $\gamma<1$, the process becomes nonstationary and $\delta=0$. If $\gamma$ approaches $1, \delta$ changes discontinuously to a value $\delta_{c}$. The order parameter of the phase transition is the limit value of the ACF. In the nonstationary phase with $c>0$ for $\gamma<1$, the memory of the history remains forever and the estimation of the PD and other parameters becomes impossible even if the length of the time series is infinite.

This study addresses the phase transition of the Pólya urn with an arbitrary memory kernel using the stochastic differential equation (SDE). The original Pólya urn is a Markov

process as the probability that the color of a newly added ball is red depends only on the 
present number of red balls in the urn. The Pólya urn with exponential decay memory kernel can also be described as a Markov process when one focuses on the probability that the color of a newly added ball is red. However, if the memory kernel is a power-law decay one, it is necessary to introduce an infinite number of auxiliary variables to make the process Markov. The auxiliary field obeys the SDE with a common Wiener process. Using the formulation, we derive the self-consistent equation for the ACF. By studying the equation, we succeed to derive the power-law exponents of the leading and subleading terms of the ACF.

Our analysis is very similar to the one that studies the Hawkes process using a stochastic differential equation[14, 15]. The Hawkes process is a non-Markov self-excited point process [16]. The Hawkes process shows a stationary-nonstationary phase transition when the branching ratio exceeds one. The critical and near-critical behaviors of the probability density function of the intensity have been studied by solving the Master equation[14].

In our study of the Pólya urn with memory kernel, we focus on the phase transition. Two universality classes are known for the phase transitions of non-linear Pólya urns [17, 18], which have the same scaling structure of order parameter as that of the absorption state phase transitions[19]. Our goal is to understand the nature of the phase transition of the "linear" Pólya urn with power-law memory kernel based on the behaviors of the order parameter. The organization of the paper is as follows. Section II defines the model. We review some results of the Pólya urn. In section III, we study the model using the SDE. We start from the exponential decay memory kernel model. The ACF decays exponentially and the process is stationary. Next we study the power-law decay memory kernel model. We introduce an auxiliary field to make the process Markovian and we derive the self-consistent equation for the ACF. Section [V] shows the results about the asymptotic behavior of the ACF for the power-law decay memory kernel model. We study the power-law exponents of the leading and sub-leading terms of the ACF. In section $\mathrm{V}$, we summarize the results.

\section{MODEL}

The Pólya urn is a binary stochastic process $X(t) \in\{0,1\}, t=1,2, \cdots$ and the probability that $X(t+1)$ takes the value 1 is

$$
P(X(t+1)=1 \mid X(s), s=1, \cdots, t)=\frac{\alpha+\sum_{s=1}^{t} X(s)}{\alpha+\beta+\sum_{s=1}^{t} 1}
$$


$\alpha, \beta>0$ are parameters and can be interpreted as the initial numbers of red (1) and blue (0) balls in the urn[13]. They are parameters and are not necessarily restricted to be integers. We denote $\alpha+\beta$ as $\theta$.

We define the Pólya urn with an arbitrary memory kernel $d(t), t=0,1, \cdots[10]$. We denote the cumulative sum of $d(t)$ as $D_{d}(t)$

$$
D_{d}(t)=\sum_{s=1}^{t} d(s-1), D_{d}(0)=0
$$

and the weighted sum of $X(t)$ with weights $d(t)$ as $S_{d}(t)$,

$$
S_{d}(t)=\sum_{s=1}^{t} X(s) d(t-s), S_{d}(0)=0
$$

$Z_{d}(t)$ is then defined as

$$
Z_{d}(t)=\frac{S_{d}(t)}{D_{d}(t)}, Z_{d}(0)=0, Z_{d}(1)=X(1)
$$

The probability that $X(t+1)$ takes the value 1 is

$$
P(X(t+1)=1 \mid X(s), s=1, \cdots, t)=\frac{\alpha+S_{d}(t)}{\theta+D_{d}(t)}=\frac{\alpha+D_{d}(t) Z_{d}(t)}{\theta+D_{d}(t)}
$$

If one chooses $d(t)=1, t=0,1, \cdots, D_{d}(t)=t$ and $S_{d}(t)=\sum_{s=1}^{t} X(s)$, so that (2) reduces to (11). The probabilistic rule of (2) is linear in $X(s)$, the expected value of $X(t)$ is $E(X(t))=$ $\alpha / \theta$.

We are interested in the response of $X(t+1)$ by the change in the initial value $X(1)$. The ACF $C(t)$ is defined as the covariance of $X(1)$ and $X(t+1)$ divided by the variance of $X(1)$ as,

$$
C(t) \equiv \frac{E(X(1) X(t+1))-(\alpha / \theta)^{2}}{V(X(1)} .
$$

We have another expression for $C(t)$ as [17],

$$
C(t)=E(X(t+1) \mid X(1)=1)-E(X(t+1) \mid X(1)=0) .
$$


$X(2)$ depends on $X(1)$ and $E(X(2) \mid X(1))=(\alpha+d(0) X(1)) /(\theta+d(0))$, we have

$$
C(1)=\frac{d(0)}{\theta+d(0)}
$$

The order parameter $c$ of the phase transition of the stochastic process is defined as the limit value of $C(t), c=\lim _{t \rightarrow \infty} C(t)$. The conditional expected value of $X(t+1)$ under the condition for $X(1)$ is the average probability that $X(t+1)$ takes the value 1 under the same condition.

$$
E(X(t+1) \mid X(1))=E(P(X(t+1)=1) \mid X(1))=\frac{\alpha+\sum_{s=1}^{t} E(X(s) \mid X(1)) d(t-s)}{\theta+D_{d}(t)} .
$$

As $C(t)=E(X(t+1) \mid X(1)=1)-E(X(t+1) \mid X(1)=0)$, we have the recursive equation for $C(t)$ as,

$$
C(t)=\frac{\sum_{s=1}^{t} C(s-1) d(t-s)}{\theta+D_{d}(t)}=\frac{d(t-1)+\sum_{s=2}^{t} C(s-1) d(t-s)}{\theta+D_{d}(t)} .
$$

One can compute $C(t), t \geq 2$ using this equation and the initial condition $C(1)=d(0) /(\theta+$ $d(0))$.

There are several choices for the memory kernel $d(t), t=0, \cdots$.

1. Pólya urn, $d(t)=1, t \geq 0$.

As we have noted before, if one choose $d(t)=1, t=0, \cdots$, the model is the Pólya urn. We denote $Z_{d}(t)$ for this case as $Z_{\infty}(t) . t Z_{\infty}(t)$ obeys a binomial distribution with shape parameters $\alpha, \beta[13]$. The expected value of $X(t)$ is $E(X(t))=\alpha / \theta . Z_{\infty}(\infty)$ obeys the beta distribution and the variance is $\frac{1}{\theta+1} \cdot \frac{\alpha \beta}{\theta^{2}}$. When one is interested in the dynamics of $X(t)$ for the initial condition $X(1)=x$, it is only necessary to alter the parameters $\alpha, \beta$ and $\theta$ as $\alpha+x, \beta+(1-x)$ and $\theta+1$, respectively. The conditional expected value $E(X(t+1) \mid X(1)=x)$ is $(\alpha+x) /(\theta+1)$ for $t \geq 1$ and $\mathrm{ACF} C(t)$ becomes constant.

$$
C(t)=\frac{1}{\theta+1}, t \geq 1
$$

The process is not stationary and not mean-reverting. The conditional variance of 
$Z_{\infty}(\infty)$ for $X(1)=x$ is

$$
V\left(Z_{\infty}(\infty) \mid X(1)=x\right)=\frac{1}{\theta+2} \cdot \frac{(\alpha+x)(\beta+(1-x))}{(\theta+1)^{2}}
$$

2. Finite memory kernel, $d(t)=1, t<r$ and $d(t)=0, t \geq r$.

There is a cutoff $r$ in the memory kernel, and $d(t)=1$ for $0 \leq t<r$, and $d(t)=0$ for $t \geq r$. For $t>r, X(t)$ is influenced by the most recent $r$ variables $X(s), s=t-$ $1, \cdots, t-r$. For $t \leq r, X(t)$ are influenced from all past variables $X(s), s=1, \cdots, t-1$. $Z_{d}(t)$ is defined as

$$
Z_{d}(t)=\left\{\begin{array}{cc}
\frac{1}{r} \sum_{s=t-r+1}^{t} X(s) & t \geq r, \\
\frac{1}{t} \sum_{s=1}^{t} X(s) & t<r .
\end{array}\right.
$$

The probability that $Z_{d}(t), t>r$ changes by $\pm 1 / r$ for the condition $Z_{d}(t)=z_{d}$ is given as

$P\left(\Delta Z_{d}(t)=+1 / r \mid Z_{d}(t)=z_{d}\right)=P\left(X(t-r+1)=0 \mid Z_{d}(t)=z_{d}\right) \cdot P\left(X(t+1)=1 \mid Z_{d}(t)=z_{d}\right)$, $P\left(\Delta Z_{d}(t)=-1 / r \mid Z_{d}(t)=z_{d}\right)=P\left(X(t-r+1)=1 \mid Z_{d}(t)=z_{d}\right) \cdot P\left(X(t+1)=0 \mid Z_{d}(t)=z_{d}\right)$.

If one adopts the mean field approximation as $P\left(X(t-r+1)=1 \mid Z_{d}(t)=z_{d}\right)=z_{d}(t)$ and $P\left(X(t-r+1)=0 \mid Z_{d}(t)=z_{d}\right)=1-z_{d}(t)$, the probabilities can be written as

$$
\begin{aligned}
& P\left(\Delta Z_{d}(t)=+1 / r \mid Z_{d}(t)=z_{d}\right)=\left(1-z_{d}\right) \cdot \frac{\alpha+r z_{d}(t)}{\theta+r} \\
& P\left(\Delta Z_{d}(t)=-1 / r \mid Z_{d}(t)=z_{d}\right)=z_{d} \cdot \frac{\beta+r\left(1-z_{d}(t)\right)}{\theta+r}
\end{aligned}
$$

By the approximation, the model becomes the Kirman's ant colony model[ [8]. In the Kirman's ant colony model, there are two food sources: 0,1 and $r$ ants. The numbers of ants that have chosen food sources 1 and 0 are $n_{1}$ and $n_{0}$, respectively. A randomly selected ant chooses food sources 1 and 0 with a probability that is linear in $n_{1}$ and $n_{0}$ among $r-1$ ants. The probability that the ratio $z=n_{1} / r$ changes $\pm 1 / r$ is given 


$$
\begin{gathered}
P\left(\Delta z=+1 \mid n_{1}(t) / r=z\right)=(1-z) \cdot \frac{\alpha+(r-1) z}{\theta+(r-1)} \\
P\left(\Delta z=-1 / r \mid n_{1}(t) / r=z\right)=z \cdot \frac{\beta+(r-1)(1-z)}{\theta+(r-1)} .
\end{gathered}
$$

The prefactors in the right hand side of the equations are the probabilities that the randomly selected ants are from food sources 0 and 1, respectively. The remaining terms are the probabilities that the selected ant chooses food sources 1 and 0 , respectively. The $\alpha, \beta$ are noises in the decision of the ants. The Pólya urn with finite memory kernel corresponds to the ant colony model in the mean filed approximation. The stationary distribution of $n_{1}$ is a beta binomial distribution, with shape parameters $\alpha, \beta$. $C(t)$ decays exponentially as $C(t) \sim \exp (-t / \xi)$, which defines the correlation length $\xi$. $\xi$ depends on the length of the memory $r$ and diverges in the limit $r \rightarrow \infty[20]$.

3. Exponential decay memory kernel, $d(t)=e^{-r t}$.

A previous study reveals that $C(t)$ decays exponentially as $C(t) \sim e^{-t / \xi}$ for $t>>1[10]$. The correlation length $\xi$ is determined by $r$,

$$
1 / \xi=\ln \left(\frac{\theta\left(1-e^{-r}\right)+1}{\theta e^{-r}\left(1-e^{-r}\right)+1}\right)>0
$$

and $\xi$ diverges in the limit $r \rightarrow 0$. In the limit $r \rightarrow \infty$, we have $d(t)=0$ for $t>0$. $X(t+1)$ depends only on $X(t)$, which corresponds to the correlated random walk $[21]$.

4. Power-law decay memory kernel, $d(t)=(1+t)^{-\gamma}$.

The model shows a phase transition at $\gamma=1[10]$. By the numerical study of the ACF $C(t)$, it was conjectured that the asymptotic behavior of $C(t)$ is

$$
C(t) \sim\left\{\begin{array}{cc}
c t^{-\gamma} & \gamma>1 \\
c t^{-\delta_{c}} & \gamma=1 \\
c t^{0} & \gamma<1
\end{array}\right.
$$

The critical power-law exponent $\delta_{c}$ at $\gamma=1$ is given as the solution of the next 
equation,

$$
\theta=\lim _{t \rightarrow \infty}\left(\int_{1 /(t+1)}^{t /(t+1)} x^{-\delta_{c}}(1-x)^{-1} d x-\ln t\right)
$$

\section{STOCHASTIC DIFFERENTIAL EQUATION}

We study the Pólya urn with memory kernel using the method of stochastic approximation [22, 23]. We start from the exponential decay memory kernel model. For the reader's convenience, we summarize the results of the analysis of the original Pólya urn using the SDE in Appendix A.

\section{A. Exponential decay memory kernel model : $d(t)=e^{-r t}$}

We denote $D_{d}(t), S_{d}(t), Z_{d}(t)$ for $d(t)=e^{-r t}$ as $D(r, t)=\sum_{s=1}^{t} e^{-r(t-s)}, S(r, t)=$ $\sum_{s=1}^{t} X(s) e^{-r(t-s)}, Z(r, t)=S(r, t) / D(r, t)$, respectively. We decompose $S(r, t+1)$ and $D(r, t+1)$ as

$$
\begin{aligned}
& D(r, t+1)=D(r, t) e^{-r}+1, \\
& S(r, t+1)=\sum_{s=1}^{t+1} X(s) e^{-r(t+1-s)}=S(r, t) e^{-r}+X(t+1) .
\end{aligned}
$$

$Z(r, t+1)$ can be decomposed as

$$
Z(r, t+1)=\frac{S(r, t+1)}{D(r, t+1)}=Z(r, t)+\frac{X(t+1)-Z(r, t)}{D(r, t+1)}
$$

For $t>>1, D(r, t)=\left(1-e^{-r t}\right) /\left(1-e^{-r}\right) \simeq 1 /\left(1-e^{-r}\right)$ and we write $D_{r}=1 /\left(1-e^{-r}\right)$. The conditional expected value and the variance of $Z(r, t+1)$ for $Z(r, t)=z_{r}$ is

$$
\begin{aligned}
E\left(Z(r, t+1) \mid Z(r, t)=z_{r}\right)-z_{r} & =\frac{E\left(X(t+1) \mid Z(r, t)=z_{r}\right)-z_{r}}{D(r, t+1)} \simeq \frac{\alpha-\theta z_{r}}{D_{r}\left(D_{r}+\theta\right)} \\
V\left(Z(r, t+1) \mid Z(r, t)=z_{r}\right) & =\frac{1}{D(r, t+1)^{2}} V\left(X(t+1) \mid Z(r, t)=z_{r}\right) \\
& \simeq \frac{1}{D_{r}^{2}} \cdot \frac{\left(\alpha+D_{r} z_{r}\right)\left(\beta+D_{r}\left(1-z_{r}\right)\right)}{\left(D_{r}+\theta\right)^{2}}
\end{aligned}
$$

Based on the assumption that ACF decays with $t$ rapidly, $Z(r, t)$ fluctuates around its mean value and $E(Z(r, t))=\alpha / \theta$, we approximate the conditional variance of $Z(r, t+1)$ for 
$Z(r, t)=z_{r}$ by replacing $z_{r}$ with $\alpha / \theta$ as,

$$
V\left(Z(r, t+1) \mid Z(r, t)=z_{r}\right) \simeq \frac{B^{2}}{D_{r}^{2}}, B^{2}=\frac{\alpha \beta}{\theta^{2}} .
$$

One reads the drift and diffusion terms from the conditional expected value and the variance, and the SDE becomes

$$
d Z(r, t)=A_{r}(\alpha-\theta Z(r, t)) d t+B_{r} d W_{t}
$$

$A_{r}, B_{r}$ are defined as,

$$
A_{r}=\frac{1}{D_{r}\left(D_{r}+\theta\right)}, B_{r}=\frac{B}{D_{r}} .
$$

It is the Ornstein-Uhlenbeck process. The condition of mean-reverting $A_{r} \theta>0$ is satisfied and $Z(r, t)$ fluctuates around $\alpha / \theta[22,24]$.

The solution for the SDE with the initial condition $Z(r, 1)=X(1)=x$ is

$$
Z(r, t)=\frac{\alpha}{\theta}\left(1-e^{-\theta A_{r}(t-1)}\right)+x \cdot e^{-\theta A_{r}(t-1)}+B_{r} e^{-\theta A_{r}(t-1)} \int_{1}^{t} e^{\theta A_{r}(s-1)} d W_{s} .
$$

The conditional expected value and the variance of $Z(r, t)$ for the initial condition $X(1)=x$ are

$$
\begin{aligned}
& E(Z(r, t) \mid X(1)=x)=\frac{\alpha}{\theta}\left(1-e^{-\theta A_{r}(t-1)}\right)+x \cdot e^{-\theta A_{r}(t-1)}, \\
& V(Z(r, t) \mid X(1)=x)=\frac{B_{r}^{2}}{2 \theta A_{r}}\left(1-e^{-\theta A_{r}(t-1)}\right) \simeq \frac{B_{r}^{2}}{2 \theta A_{r}} .
\end{aligned}
$$

The conditional variance does not depend on the initial condition. For $r<<1, D_{r} \simeq 1 / r$, $A_{r} \simeq 1 / D_{r}^{2}=r^{2}, B_{r}^{2} \simeq r^{2} B^{2}$ and $V(Z(r, t) \mid X(1)=x) \simeq B^{2} /(2 \theta)$. The ACF is estimated as

$$
\begin{aligned}
C(t) & =E(X(t+1) \mid X(1)=1)-E(X(t+1) \mid X(1)=0) \\
& =E(P(X(t+1)=1) \mid X(1)=1)-E(P(X(t+1)=1) \mid X(1)=0) \\
& =\frac{D_{r} E(Z(r, t) \mid Z(1)=1)+\alpha}{D_{r}+\theta}-\frac{D_{r} E(Z(r, t) \mid X(1)=0)+\alpha}{D_{r}+\theta} \\
& =\frac{D_{r}}{D_{r}+\theta} e^{-\theta A_{r}(t-1)}=\frac{D_{r}}{D_{r}+\theta} e^{-\frac{\theta}{\left(D_{r}+\theta\right) D_{r}} t} .
\end{aligned}
$$

$C(t)$ decays exponentially and $C(t) \propto e^{-\theta r^{2} t}$ for $r<<1$. $Z(r, t)$ forgets the initial value $X(1)$ 
and fluctuates around $\alpha / \theta$ with variance $B^{2} /(2 \theta)$ for $r<<1$. In the limit $r \rightarrow 0, D(r, t) \rightarrow t$, and $C(t)$ becomes constant. The stochastic process remembers its initial condition $X(1)$ and the process is not stationary. A discontinuous phase transition occurs at $r=0$.

\section{B. Power-law decay memory kernel model : $d(t)=(t+1)^{-\gamma}$}

We denote $D_{d}(t), S_{d}(t), Z_{d}(t)$ for $d(t)=(t+1)^{-\gamma}$ as $D_{\gamma}(t)=\sum_{s=1}^{t}(t-s+1)^{-\gamma}, S_{\gamma}(t)=$ $\sum_{s=1}^{t} X(s)(t-s+1)^{-\gamma}, Z_{\gamma}(t)=S_{\gamma}(t) / D_{\gamma}(t)$, respectively. Different from the exponential decay memory kernel model, it is impossible to decompose $Z_{\gamma}(t+1)$ with $Z_{\gamma}(t)$ and $X(t+1)$

directly. We introduce an auxiliary field $\{Z(r, t), 0<r<\infty\}$ and express $Z_{\gamma}(t)$ as the superposition of the field. Using the decomposition of $Z(r, t+1)$ with $Z(r, t)$ and $X(t+1)$ of (7), we derive the SDE for the auxiliary field $Z(r, t)$ and estimate $Z_{\gamma}(t)$.

First, we express $(t+1)^{-\gamma}$ as the superposition of $e^{-r t}$ with the gamma distribution $f_{\gamma}(r)=r^{\gamma-1} e^{-r} / \Gamma(\gamma)$ as

$$
(t+1)^{-\gamma}=\int_{0}^{\infty} e^{-r t} \cdot f_{\gamma}(r) d r .
$$

We also express $D_{\gamma}(t)$ and $S_{\gamma}(t)$ as the superposition of $D(r, t)$ and $S(r, t)$ with $f_{\gamma}(r)$.

$$
\begin{aligned}
& D_{\gamma}(t)=\int_{0}^{\infty} D(r, t) f_{\gamma}(r) d r \\
& S_{\gamma}(t)=\int_{0}^{\infty} S(r, t) f_{\gamma}(r) d r=\int_{0}^{\infty} D(r, t) Z(r, t) f_{\gamma}(r) d r
\end{aligned}
$$

$Z_{\gamma}(t)$ is then written as

$$
Z_{\gamma}(t)=\frac{S_{\gamma}(t)}{D_{\gamma}(t)}=\frac{\int_{0}^{\infty} Z(r, t) D(r, t) f_{\gamma}(r) d r}{\int_{0}^{\infty} D(r, t) f_{\gamma}(r) d r}
$$

We denote the average of function $A(r, t)$ with the weight $D(r, t) f_{\gamma}(r)$ by $<A(r, t)>$,

$$
<A(r, t)>\equiv \frac{\int_{0}^{\infty} A(r, t) D(r, t) f_{\gamma}(r) d r}{D_{\gamma}(t)}
$$

The denominator ensures the identity $<1>=1$ and $Z_{\gamma}(t)$ is written as $\langle Z(r, t)\rangle$.

Next we derive the SDE for the auxiliary field $\{Z(r, t), 0<r<\infty\}$. When $\{Z(r, t)=$ $z(r), 0<r<\infty\}, Z_{\gamma}(t)$ is estimated as $Z_{\gamma}(t)=z_{\gamma} \equiv<z(r)>$. The probability that $X(t+1)$ 
takes the value 1 with the condition $\{Z(r, t)=z(r), 0<r<\infty\}$ is then given as

$$
P(X(t+1)=1 \mid\{Z(r, t)=z(r), 0<r<\infty\})=\frac{\alpha+D_{\gamma}(t)<z(r)>}{\theta+D_{\gamma}(t)}=\frac{\alpha+D_{\gamma}(t) z_{\gamma}}{\theta+D_{\gamma}(t)}
$$

As $Z(r, t+1)=Z(r, t)+\frac{X(t+1)-Z(r, t)}{D(r, t+1)}$ in (7), we have,

$$
\begin{aligned}
& E(Z(r, t+1) \mid\{Z(r, t)=z(r), 0<r<\infty\})=z(r)+\frac{E\left(X(t+1) \mid Z_{\gamma}(t)=z_{\gamma}\right)-z(r)}{D(r, t+1)} \\
= & z(r)+\frac{\theta z_{0}+D_{\gamma}(t)<z(r)>-\left(\theta+D_{\gamma}(t)\right) z(r)}{\left(\theta+D_{\gamma}(t)\right) D(r, t+1)} .
\end{aligned}
$$

Here, we define $z_{0}=\alpha / \theta$. We denote the weighted average of $z_{0}$ and $z_{\gamma}(t)=<z(r, t)>$ with the weights $\theta$ and $D_{\gamma}(t)$ as $z(t)$,

$$
z(t) \equiv \frac{\theta z_{0}+D_{\gamma}(t) z_{\gamma}(t)}{\theta+D_{\gamma}(t)}=\frac{\theta z_{0}+D_{\gamma}(t)<z(r, t)>}{\theta+D_{\gamma}(t)}
$$

Then, the conditional expected value is written as

$$
E(Z(r, t+1) \mid\{Z(r, t)=z(r), 0<r<\infty\})=z(r)+\frac{z(t)-z(r)}{D_{r}(t+1)}
$$

Likewise, we estimate the conditional variance as

$$
\begin{aligned}
& V\left(Z(r, t+1) \mid\left\{Z_{r}(t)=z_{r}, 0<r<\infty\right\}\right)=\frac{V(X(t+1) \mid\{Z(r, t)=z(r), 0<r<\infty\})}{D_{r}(t+1)^{2}} \\
= & \frac{\left(D_{\gamma}(t) z_{\gamma}+\theta z_{0}\right)\left(D_{\gamma}(t)\left(1-z_{\gamma}\right)+\theta\left(1-z_{0}\right)\right)}{D_{r}(t+1)^{2}\left(D_{\gamma}(t+1)+\theta\right)^{2}}=\frac{z(t)(1-z(t))}{D_{r}(t+1)^{2}} .
\end{aligned}
$$

We assume that $\mathrm{z}(\mathrm{t})$ does not converge to 0 nor 1 and the numerator of the variance does not vanish. We can replace the numerator of the variance with constant $B^{2}$ for simplicity without affecting the essence of the process. One reads the drift and the diffusion terms and the SDE for the auxiliary field $\{Z(r, t), 0<r<\infty\}$ becomes

$$
d Z(r, t)=\frac{Z(t)-Z(r, t)}{D_{r}(t)}+\frac{B}{D_{r}(t)} d W_{t}, 0<r<\infty .
$$

We note that the auxiliary field $\{Z(r, t), 0<r<\infty\}$ shares the common Wiener process $W_{t}$ as it emerges from $X(t+1)$.

The "formal" solution for the auxiliary field $\{Z(r, t)\}$ to the initial value problem 
$\{Z(r, 1)=X(1)=x, 0<r<\infty\}$ is

$Z(r, t)=I(r, t) \cdot x+I(r, t) \int_{1}^{t} \frac{Z(s)}{D(r, s)} I(r, s)^{-1} d s+I(r, t) \int_{0}^{t} \frac{B}{D(r, s)} I(r, s)^{-1} d W_{s}, 0<r<\infty$.

Here, $I(r, t)$ is defined as

$$
I(r, t) \equiv \exp \left(-\int_{1}^{t} \frac{1}{D(r, s)} d s\right)=\left(\frac{e^{r t}-1}{e^{r}-1}\right)^{-\frac{1-e^{-r}}{r}}
$$

The reason to call the solution "formal" is that $Z(s)$ in the integral of the second term of the solution is calculated using $\{Z(r, t), 0<r<\infty\}$. We consider the deviation of $Z(r, t)$ from $z_{0}$ as $\Delta Z(r, t)$, i.e., $Z(r, t)=z_{0}+\Delta Z(r, t) . \Delta Z_{\gamma}(t)$ is also defined as $\Delta Z_{\gamma}(t)=<$ $\Delta Z(r, t)>=<Z(r, t)-z_{0}>=Z_{\gamma}(t)-z_{0} . Z(t)$ is then written as

$$
Z(t)=z_{0}+\frac{D_{\gamma}(t) \Delta Z_{\gamma}(t)}{\theta+D_{\gamma}(t)}
$$

The formal solution for $\{Z(r, t)\}$ becomes

$$
\begin{aligned}
Z(r, t) & =I(r, t) \cdot x+(1-I(r, t)) \cdot z_{0} \\
& +I(r, t)\left(\int_{1}^{t} \frac{1}{D(r, s)}\left(\frac{D_{\gamma}(s) \Delta z_{\gamma}(s)}{\theta+D_{\gamma}(s)}\right) I(r, s)^{-1} d s+\int_{0}^{t} \frac{B}{D(r, s)} I(r, s)^{-1} d W_{s}\right), 0<r<\infty
\end{aligned}
$$

$Z_{\gamma}(t)=<Z(r, t)>$ is then estimated as

$$
\begin{aligned}
Z_{\gamma}(t) & =z_{0}+<I_{r}(t)>\left(x-z_{0}\right) \\
& +\left\langle I(r, t)\left(\int_{1}^{t} \frac{1}{D(r, s)}\left(\frac{D_{\gamma}(s) \Delta Z_{\gamma}(s)}{\theta+D_{\gamma}(s)}\right) I(r, s)^{-1} d s+\int_{0}^{t} \frac{B}{D(r, s)} I(r, s)^{-1} d W_{s}\right)\right\rangle .
\end{aligned}
$$

To estimate the average $<>$ in the right hand side of the solution for $Z_{\gamma}(t)$, we derive a useful approximation integral formula for the arbitrary function $f(r)$, which is valid for large $t$.

$$
\int_{0}^{\infty} f(r) f_{\gamma}(r) e^{-r t} d r=\int_{0}^{1 /(t+1)} f(r) \cdot \gamma r^{\gamma-1} d r
$$

It replaces the definite integral of $f(r)$ multiplied by $f_{\gamma}(r) e^{-r t}$ over $r \in[0, \infty)$ with the definite integral of $f(r)$ multiplied by $\gamma r^{\gamma-1}$ over $r \in[0,1 /(t+1)]$. For large $t, e^{-r t}<<1$ for $r>>1 / t$ and $f(r)$ with $r \sim 0$ dominates the asymptotic behavior. In the limit $r \rightarrow 0, f_{\gamma}(r)$ 
can be replaced with $\gamma r^{\gamma-1}$. Here, we adopt $\gamma r^{\gamma-1}$ instead of $r^{\gamma-1} / \Gamma(\gamma)$ to ensure that the approximation formula gives $(t+1)^{-\gamma}$ for $f(r)=1$, which is the identity of (8)). The formula is useful to study the asymptotic (large $t$ ) behavior of the left-hand side of the equation.

Then, we have two approximation formulas,

$$
\begin{aligned}
<I_{r}(t)> & \simeq \frac{t^{-\gamma}}{D_{\gamma}(t)} \\
<I_{r}(t) \frac{1}{D_{r}(s)} I_{r}(s)^{-1}> & \simeq \frac{(t-s+1)^{-\gamma}}{D_{\gamma}(t)} .
\end{aligned}
$$

Using the results, we have

$$
\begin{aligned}
Z_{\gamma}(t) & =z_{0}+\frac{t^{-\gamma}}{D_{\gamma}(t)}\left(x-z_{0}\right) \\
& +\frac{1}{D_{\gamma}(t)}\left(\int_{1}^{t}(t-s+1)^{-\gamma}\left(\frac{D_{\gamma}(s) \Delta z_{\gamma}(s)}{\theta+D_{\gamma}(s)}\right) d s+\int_{1}^{t}(t-s+1)^{-\gamma} B d W_{s}\right) .
\end{aligned}
$$

The conditional expected value of $Z_{\gamma}(t)$ for $X(1)=x$ is

$$
\begin{aligned}
E\left(Z_{\gamma}(t) \mid X(1)=x\right) & =z_{0}+\frac{t^{-\gamma}}{D_{\gamma}(t)}\left(x-z_{0}\right) \\
& +\frac{1}{D_{\gamma}(t)}\left(\int_{1}^{t}(t-s+1)^{-\gamma}\left(\frac{D_{\gamma}(s)\left(E\left(Z_{\gamma}(s) \mid X(1)=x\right)-z_{0}\right)}{\theta+D_{\gamma}(s)}\right) d s\right) .
\end{aligned}
$$

$C(t)$ is then given as

$$
C(t)=\frac{1}{D_{\gamma}(t)+\theta}\left(t^{-\gamma}+\int_{1}^{t}(t-s+1)^{-\gamma} C(s)\right) .
$$

We derive the integral equation for $C(t)$, which is the continuous approximation of (3).

\section{ASYMPTOTIC BEHAVIOR OF CORRELATION FUNCTION}

We study the asymptotic behavior of the ACF for the power-law decay memory kernel model. We assume the power-law asymptotic behavior of the leading and sub-leading terms of the $\mathrm{ACF}$ as

$$
C(t)=c t^{-\delta}+c^{\prime} t^{-\delta^{\prime}}, \delta^{\prime}>\delta \text { for } t>>1
$$


We multiply $\left(D_{\gamma}(t)+\theta\right)$ on both sides of (12) and we obtain

$$
\left(D_{\gamma}(t)+\theta\right) C(t)=\left(t^{-\gamma}+\int_{1}^{t}(t-s+1)^{-\gamma} C(s) d s\right) .
$$

We substitute (13) into (12) and compare the power-law exponent of the leading and subleading terms on both sides of the equation.

The asymptotic behaviors of $D_{\gamma}(t)$ are $1 /(\gamma-1), \ln t$ and $t^{1-\gamma} /(1-\gamma)$ for $\gamma>1,=1$ and $<1$, respectively. We put $c t^{-\delta}+c^{\prime} t^{-\delta^{\prime}}$ in $C(t)$ and estimate the integral of the right hand side of (14).

$$
\int_{1}^{t}(t-s+1)^{-\gamma}\left(c s^{-\delta}+c s^{-\delta^{\prime}}\right) d s
$$

After changing the variable from $s$ to $x=s /(t+1)$, the first term of the integral becomes

$$
c(t+1)^{1-\delta-\gamma} \int_{\epsilon}^{1-\epsilon}(1-x)^{-\gamma} x^{-\delta} d x
$$

Here we write $\epsilon=1 /(t+1)$. If the exponents $\gamma, \delta$ of $x, 1-x$ in the integrand are smaller than 1 , the integral converges to the beta function, $B(1-\gamma, 1-\delta)$, in the limit $\epsilon \rightarrow 0$. If $\gamma \geq 1$ or $\delta \geq 1$, it is necessary to regularize the integral. We denote the integral as $B(1-\gamma, 1-\delta, \epsilon)$,

$$
B(1-\gamma, 1-\delta, \epsilon) \equiv \int_{\epsilon}^{1-\epsilon}(1-x)^{-\gamma} x^{-\delta} d x
$$

We write the finite term of $B(1-\gamma, 1-\delta, \epsilon)$ in the limit $\epsilon$ as $B_{r e g}(1-\gamma, 1-\delta)$.

$$
B(1-\gamma, 1-\delta, \epsilon)=B_{\text {reg }}(1-\gamma, 1-\delta)+\text { divergent terms }
$$

When $\gamma<1, \delta<1$, the integral converges and $B_{\text {reg }}(1-\gamma, 1-\delta)$ is $B(1-\gamma, 1-\delta)$.

The regularization procedure is simple. When $\gamma>1$ and $\delta<1$, the integral of $(1-x)^{-\gamma} x^{-\delta}$ diverges when the upper bound $1-\epsilon$ goes to 1 . We regularize the integral as

$$
\begin{aligned}
B(1-\gamma, 1-\delta, \epsilon) & =\int_{1 / \epsilon}^{1-\epsilon}(1-x)^{-\gamma}\left(x^{-\delta}-1\right) d x+\int_{1 / \epsilon}^{1-\epsilon}(1-x)^{-\gamma} d x \\
& =B_{r e g}(1-\gamma, 1-\delta, \epsilon)+\frac{1}{\gamma-1} \epsilon^{1-\gamma} .
\end{aligned}
$$

Here we neglect a term that vanishes in the limit $\epsilon \rightarrow 0$. The second term diverges as 
$\epsilon^{1-\gamma} \propto t^{\gamma-1}$. Likewise for $\gamma>1, \delta>1$, the divergence of the integral of $(1-x)^{-\gamma} x^{-\delta}$ comes from both bounds of the integral. We regularize the integral as

$$
\begin{aligned}
B(1-\gamma, 1-\delta, \epsilon) & =\int_{1 / \epsilon}^{1-\epsilon}\left(\left((1-x)^{-\gamma}-1\right)\left(x^{- \text {delta }}-1\right)-1\right) d x+\int_{1 / \epsilon}^{1-\epsilon}(1-x)^{-\gamma} d x+\int_{1 / \epsilon}^{1-\epsilon} x^{-\delta} d x \\
& =B_{r e g}(1-\gamma, 1-\delta, \epsilon)+\frac{1}{\gamma-1} \epsilon^{1-\gamma}+\frac{1}{\delta-1} \epsilon^{1-\delta}
\end{aligned}
$$

When $\gamma=1$ and $\delta<1$, a logarithmic divergence appears, and we have

$$
\begin{aligned}
B(0,1-\delta, \epsilon) & =\int_{1 / \epsilon}^{1-\epsilon}\left((1-x)^{-1}\right)\left(x^{- \text {delta }}-1\right) d x+\int_{1 / \epsilon}^{1-\epsilon}(1-x)^{-1} d x \\
& =B_{r e g}(0,1-\delta, \epsilon)-\ln \epsilon
\end{aligned}
$$

Using $B(1-\gamma, 1-\delta), B\left(1-\gamma, 1-\delta^{\prime}\right)$, (14) becomes

$$
\left(D_{\gamma}(t)+\theta\right)\left(c t^{-\delta}+c^{\prime} t^{-\delta^{\prime}}\right)=t^{-\gamma}+t^{1-\gamma-\delta} B(1-\gamma, 1-\delta, \epsilon)+t^{1-\gamma-\delta^{\prime}} B\left(1-\gamma, 1-\delta^{\prime}, \epsilon\right) .
$$

1. $\gamma<1$ case.

$D_{\gamma}(t)=t^{1-\gamma} /(1-\gamma)$ and we assume $\delta<1$. (15) becomes,

$$
\begin{aligned}
\left(\theta+t^{1-\gamma} /(1-\gamma)\right)\left(c t^{-\delta}+c^{\prime} t^{-\delta^{\prime}}\right) & =t^{-\gamma}+c t^{1-\gamma-\delta} B(1-\gamma, 1-\delta) \\
& +c^{\prime} t^{1-\gamma-\delta^{\prime}}\left(B_{r e g}\left(1-\gamma, 1-\delta^{\prime}\right)+\frac{1}{\delta^{\prime}-1} t^{\delta^{\prime}-1}\right)
\end{aligned}
$$

The last term $\frac{1}{\delta^{\prime}-1} t^{\delta^{\prime}-1}$ does not appear when $\delta^{\prime}<1$. The leading term of the left-hand side is $c /(1-\gamma) t^{1-\gamma-\delta}$. The leading term of the right-hand side is $c B(1-\gamma, 1-\delta) t^{1-\gamma-\delta}$ as $1-\gamma-\delta>1-\gamma-\delta^{\prime}$ and $1-\gamma-\delta>-\gamma$. By equating the coefficients of the leading terms, we obtain an identity

$$
\frac{1}{1-\gamma}=B(1-\gamma, 1-\delta)
$$

As $B(1-\gamma, 1)=1 /(1-\gamma)$, we obtain the result $\delta=0$. The subleading term of the left-hand side is $O\left(t^{0}\right)$ as $\delta=0$, we have $1-\gamma-\delta^{\prime}=0$. We obtain $\delta^{\prime}=1-\gamma$. If one assume $\delta>1$, we cannot match $O\left(t^{0}\right)$ terms of both sides of (15). We can exclude the possibility $\delta, \delta^{\prime}>1$ for $\gamma<1$. 
2. $\gamma=1$ case.

$D_{\gamma}(t)=\ln t$ and we assume $\delta<1$. (15) becomes,

$(\theta+\ln t)\left(c t^{-\delta}+c^{\prime} t^{-\delta^{\prime}}\right)=t^{-1}+c t^{-\delta}\left(B_{r e g}(0,1-\delta)+\ln t\right)+c^{\prime} t^{-\delta^{\prime}}\left(B_{r e g}\left(0,1-\delta^{\prime}\right)+\ln t+\frac{1}{\delta^{\prime}-1} t^{\delta^{\prime}-1}\right)$.

The leading term is $O\left(\ln t \cdot t^{-\delta}\right)$ and we obtain a trivial identity $c=c$. The subleading term is $O\left(t^{-\delta}\right)$ and we obtain the identity

$$
\theta=B_{\text {reg }}(0,1-\delta)
$$

By solving the identity, we estimate $\delta_{c}$, the critical exponent of the ACF, $C(t) \propto t^{-\delta_{c}}$. The estimation of $\delta^{\prime}$ is obscure, as t The same identity with $\delta$ holds for $\delta^{\prime}$ by comparing the $O\left(t^{-\delta^{\prime}}\right)$ terms. It suggests that $\delta^{\prime}=\delta$ at $\gamma=1$.

3. $\gamma>1$ case.

$D_{\gamma}(t)=1 /(\gamma-1)$ and we assume $1<\delta<\delta^{\prime}$. (15) becomes,

$$
\begin{aligned}
(\theta+1 /(\gamma-1))\left(c t^{-\delta}+c^{\prime} t^{-\delta^{\prime}}\right) & =t^{-1}+c t^{1-\gamma-\delta}\left(B_{\text {reg }}(1-\gamma, 1-\delta)+\frac{1}{\gamma-1} t^{\gamma-1}+\frac{1}{\delta-1} t^{\delta-1}\right) \\
& +c^{\prime} t^{1-\gamma-\delta^{\prime}}\left(B_{r e g}\left(1-\gamma, 1-\delta^{\prime}\right)+\frac{1}{\gamma-1} t^{\gamma-1}+\frac{1}{\delta^{\prime}-1} t^{\delta^{\prime}-1}\right) .
\end{aligned}
$$

The leading term is $O\left(t^{-\delta}\right)$ and we obtain $\delta=\gamma$. The subleading term is $O\left(t^{-\delta^{\prime}}\right)$ and we obtain $\delta^{\prime}=2 \gamma-1$.

The power-law exponents of the leading and subleading terms of the ACF $C(t)$ are summarized as

$$
\left(\delta, \delta^{\prime}\right)=\left\{\begin{array}{cc}
(\gamma, 2 \gamma-1) & \gamma>1 \\
\left(\delta_{c}, \delta_{c}\right) & \gamma=1 \\
(0,1-\gamma) & \gamma<1
\end{array}\right.
$$

We denote the values by $\left(\delta_{t h}, \delta_{t h}^{\prime}\right)$ to distinguish them from the numerically estimated values, which are denoted as $\left(\delta_{\text {num }}, \delta_{\text {num }}^{\prime}\right)$.

We solve the recursive relation of (3) numerically and estimate $C(t)$ for $t \leq 2^{8} \times 10^{4}$. We set $(\alpha, \beta)=(1,1)$ and $\gamma \in[0.0,0.1,0,2, \cdots, 2.0]$. For large $t>>1$, the leading term of $C(t)$ 
is $c t^{-\delta}$, and we have the relation for $\delta$,

$$
\delta=\lim _{t \rightarrow \infty} \ln _{2} C(t) / C(2 t)
$$

We estimate $\delta_{\text {num }}$ by the formula with $t=2^{7} \times 10^{4}$. About $\delta_{\text {num }}^{\prime}$, we use the relation

$$
\delta^{\prime}=\lim _{t \rightarrow \infty} \ln _{2} \frac{C(t)-C(2 t) 2^{\delta}}{C(2 t)-C(4 t) 2^{\delta}}
$$

The relation is based on the relation for the subleading term of $C(t), C(t)-C(2 t) 2^{\delta} \simeq$ $c^{\prime} t^{-\delta^{\prime}}\left(1-2^{\delta-\delta^{\prime}}\right)$ for $t>>1$. We estimate $\delta_{\text {num }}^{\prime}$ using the formula $t=2^{6} \times 10^{4}$.

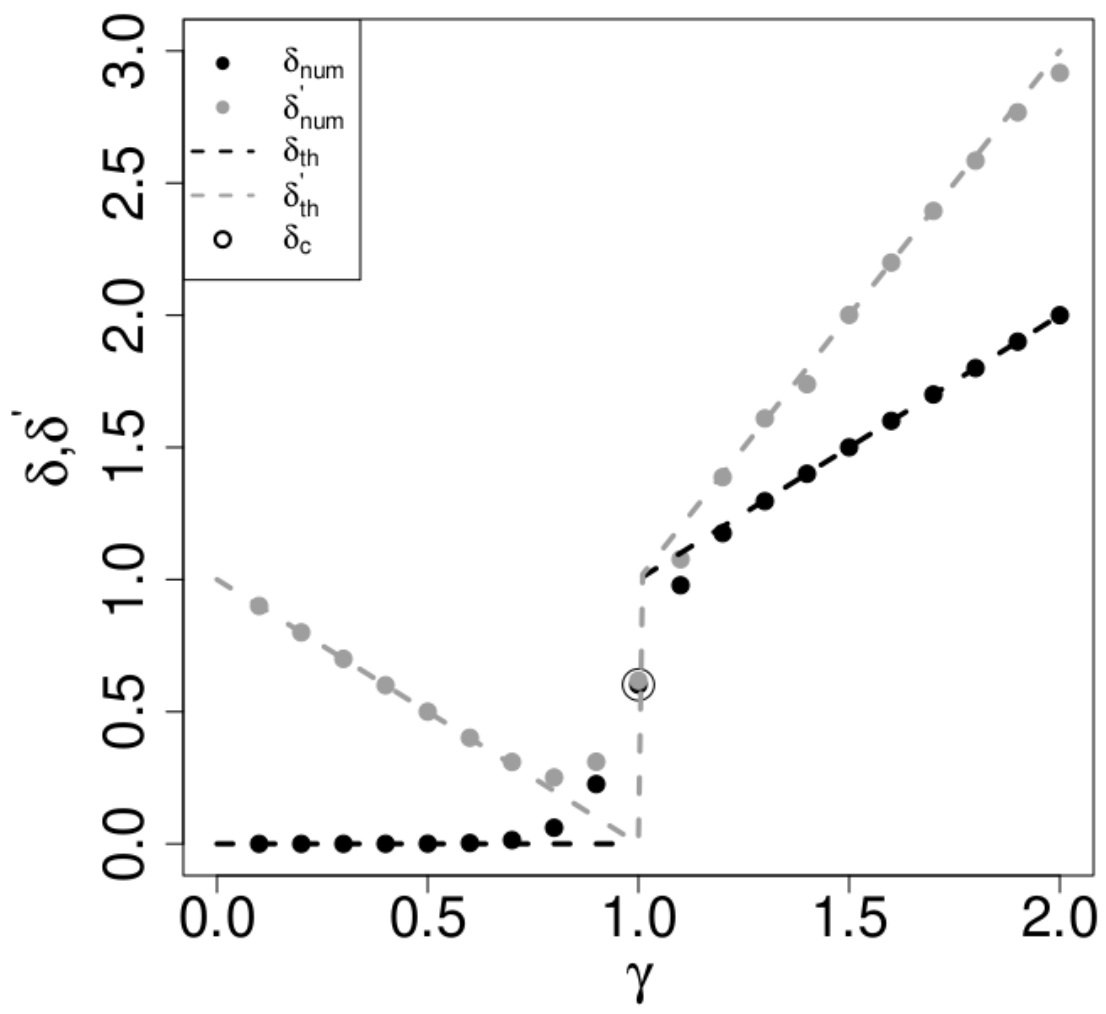

FIG. 1. Plot of $\left(\delta_{\text {num }}, \delta_{\text {num }}^{\prime}\right)$ vs. $\gamma$. We estimate the power-law exponents using (18) and (19). $\left(\delta_{t h}, \delta_{t h}^{\prime}\right)$ are the theoretically predicted values in (17).

Figure 1 shows $\left(\delta_{\text {num }}, \delta_{\text {num }}^{\prime}\right)$ vs. $\gamma$. For reference, we also plot $\left(\delta_{t h}, \delta_{t h}^{\prime}\right)$. As can be clearly observed, if $\gamma$ is far from the critical value $\gamma_{c}=1$, the numerically estimated values agree 
with the theoretical results. However, near the critical point, the discrepancy becomes large. At the critical point, both $\delta_{\text {num }}, \delta_{\text {num }}^{\prime}$ coincide with $\delta_{c}$.

\section{CONCLUSION}

We study the Pólya urn with an arbitrary memory kernel. Previously, we have shown that the process shows a phase transition for the power-law decay memory kernel[10]. We formulate the problem using the method of stochastic approximation. It is necessary to introduce an auxiliary field $\{Z(r, t), 0<r<\infty\}$ to reduce the process Markovian. The SDE for the auxiliary field are coupled as the field is driven by a common Wiener process. We obtain the formal solution for the initial value problem and derive the self-consistent equation for the $\mathrm{ACF}$ in the framework. We estimate the power-law exponents for the leading and subleading terms of the ACF. The results for the power-law exponents are new except for $\delta_{c}$ at the critical point.

The essential difference from the continuous phase transitions of the nonlinear Pólya urn is the lack of the universal function. The transition of nonlinear Pólya urns has the correlation length scale $\xi$ for the decay of the ACF and the asymptotic behavior of the ACF obeys a scaling relation. For the existence of the scaling relation for ACF, the continuity of the power-law exponent and the logarithmic behavior of the ACF at the critical point is crucial [17, 18]. In the phase transition of the Pólya urn with power-law decay, the powerlaw exponent is discontinuous at $\gamma=1$ and the ACF does not show logarithmic behavior at the critical point. The phase transition of the Pólya urn with power-law memory kernel is completely different from that of the nonlinear Pólya urn. The nature of the former is the phase transition of the stationary-nonstationary phase transition. The nature of the latter is the absorption-state phase transition where there exists the scaling relation for the order parameter.

This study also shows the importance of the correlation of the Wiener process. The auxiliary field $\{Z(r, t), 0<r<\infty\}$, which is introduced for the Pólya urn with power-law decay memory kernel, shares the same Wiener process $W_{t}$ and it induces the coupling among the modes of the field. If $\{Z(r, t), 0<r<\infty\}$ are driven by independent Wiener processes $\{W(r, t), 0<r<\infty\}$, they obey the Ornstein-Uhlenbeck process for the exponential decay memory kernel independently from each other. They are mean-reverting and converge to 
the same equilibrium $\alpha / \theta$. Even though $Z(r, t)$ with small $r$ should have long relaxation time, $Z_{\gamma}(t)=<Z(r, t)>$ also converges to the same equilibrium. The phase transition does not occur in the case. The condition of the mean-revering for the multivariate OrnsteinUhlenbeck process is known [24]. By the correlation of the Wiener process, the condition should be broken. To understand the importance of the correlation of the Wiener process for the asymptotic behavior of the process, we need to solve the initial value problem for the SDE of the coupled auxiliary field.

[1] R. N. Mantegna and H. E. Stanley, Introduction to Econophysics: Correlations and Complexity in Finance (Cambridge University Press, Cambridge, 2007).

[2] S. Galam, Int. J. Mod. Phys. C 19, 409 (2008).

[3] C. Castellano, S. Fortunato, and V. Loreto, Rev.Mod.Phys. 81, 591 (2009).

[4] W. B. Arthur, Econ. Jour. 99, 116 (1989).

[5] S. Bikhchandani, D. Hirshleifer, and I. Welch, J. Polit. Econ. 100, 992 (1992).

[6] R. Cont and J. Bouchaud, Macroecon. Dynam. 4, 170 (2000).

[7] S. Mori, K. Nakayama, and M. Hisakado, Phys.Rev. E 94, 052301 (2016).

[8] A. Kirman, Q. J. Econ. 108, 137 (1993).

[9] T. Lux, Econ. J. 105, 881 (1995).

[10] M. Hisakado and S. Mori, Physica A 544, 123480 (2020).

[11] K. Pluto and D. Tasche, in The Basel II Risk Parameters, edited by B.Engelmann. and R. Rauhmeier (Springer, Berlin, 2011) pp. 75-101.

[12] M. Hisakado and S. Mori, Physica A 563, 125435 (2021).

[13] G.Pólya, Ann. Inst. Henri Poincaré 1, 117 (1931).

[14] K. Kanazawa and D.Sornette, Phys. Rev. Lett. 125, 138301 (2020).

[15] K. Kanazawa and D.Sornette, Phys. Rev. Research. 2, 033442 (2020).

[16] A. Hawkes, J. R. Stat. Soc. Ser. B 33, 438 (1971).

[17] S. Mori and M. Hisakado, Phys.Rev. E 92, 052112 (2015).

[18] K. Nakayama and S. Mori, Phys. Rev.E 104, 014109 (2021).

[19] H. Hinrichsen, Adv.Phys. 49, 815 (2000).

[20] S. Mori and M. Hisakado, J.Phys.Soc.Jpn. 84, 054001 (2015). 
[21] W. Böhm, J. Appl. Prob. 37, 470 (2000).

[22] C. Gardiner, Stochastic Methods: A handbook for the Natural and Social Science, 4th ed. (Springer, Berlin, 2009).

[23] H. Renlund, "Generalized pólya urns via stochastic approximation," arXiv:1002.3716 [math.PR].

[24] P. Vatiwutipong and N. Phewchean, Adv.Differ.Equ. , 276 (2019).

\section{Appendix A: SDE analysis of Pólya urn}

We study the Pólya urn using the SDE for the reader's convenience. We denote $D_{d}(t), S_{d}(t), Z_{d}(t)$ for the infinite memory kernel as $D_{\infty}(t)=t, S_{\infty}(t)=\sum_{s=1}^{t} X(s), Z_{\infty}(t)=$ $\sum_{s=1}^{t} X(s) / t$, respectively. To estimate the expected value and the variance of $Z_{\infty}(t+1)$ with the condition $Z_{\infty}(t)=z$, we decompose $Z_{\infty}(t+1)$ as

$$
Z_{\infty}(t+1)=Z_{\infty}(t)+\frac{1}{t+1}\left(X(t)-Z_{\infty}(t)\right)
$$

We estimate the conditional expected value and conditional variance of $Z_{\infty}(t+1)$ as follows:

$$
\begin{aligned}
E\left(Z_{\infty}(t+1) \mid Z_{\infty}(t)=z\right)-z & =\frac{\alpha-\theta z}{\left(D_{\infty}(t)+\theta\right) D_{\infty}(t+1)} \\
V\left(Z_{\infty}(t+1) \mid Z_{\infty}(t)=z\right) & =\frac{1}{D_{\infty}(t+1)^{2}} \cdot \frac{\alpha+t z}{\theta+t} \cdot \frac{\beta+t(1-z)}{\theta+t} .
\end{aligned}
$$

We read the drift $A_{\infty}(t)$ and variance $B_{\infty}(t)$ from them and derive the SDE [22] as

$$
d Z_{\infty}=A_{\infty}\left(\alpha-\theta Z_{\infty}\right) d t+B_{\infty} d W_{t}
$$

$A_{\infty}(t), B_{\infty}(t)$ are defined as follows:

$$
A_{\infty}(t)=\frac{1}{D_{\infty}(t+1)\left(\theta+D_{\infty}(t)\right)}, B_{\infty}(t)=\frac{B}{D_{\infty}(t+1)}, B=\sqrt{\frac{(\alpha+t z)(\beta+t(1-z))}{(\theta+t)^{2}}} .
$$

As $E\left(Z_{\infty}(t) \mid X(1)=x\right)=(\alpha+x) /(\theta+1)$ for the Pólya urn, we take the limit $t \rightarrow \infty$ by 
replacing $z$ with $(\alpha+x) /(\theta+1)$ and approximate $B$ as

$$
B=\sqrt{\frac{(\alpha+x)(\beta+(1-x))}{(\theta+1)^{2}}}
$$

The SDE is the Ornstein-Uhlenbeck type SDE[22]. The solution with the initial condition $Z_{\infty}(1)=X(1)=x$ is

$$
Z_{\infty}(t)=\frac{\alpha}{\theta}\left(1-e^{-\theta \int_{1}^{t} A_{\infty}(s) d s}\right)+x \cdot e^{-\theta \int_{1}^{t} A_{\infty}(s) d s}+e^{-\theta \int_{1}^{t} A_{\infty}(s) d s} \int_{1}^{t} B_{\infty}(s) e^{\theta \int_{1}^{s} A_{\infty}(u) d u} d W_{s} .
$$

We have $\int_{1}^{t} A_{\infty}(s) d s=\frac{1}{\theta} \ln \frac{t(1+\theta)}{t+\theta}$, and the solution is written as follows:

$$
Z_{\infty}(t)=\frac{\alpha}{\theta}\left(1-\frac{t+\theta}{t(1+\theta)}\right)+x \cdot \frac{t+\theta}{t(1+\theta)}+\left(1+\frac{\theta}{t}\right) \int_{1}^{t} \frac{B}{1+s} \cdot \frac{s}{s+\theta} d W_{s}
$$

The conditional expected value of $Z_{\infty}(t)$ for $X(1)=x$ is

$$
E\left(Z_{\infty}(t) \mid X(1)=x\right)=\frac{\alpha}{\theta}\left(1-\frac{t+\theta}{t(1+\theta)}\right)+x \cdot \frac{t+\theta}{t(1+\theta)} \stackrel{t \gg 1}{=} \frac{\alpha}{\theta} \cdot \frac{\theta}{1+\theta}+x \cdot \frac{1}{1+\theta} .
$$

The ACF is then estimated as

$$
\begin{aligned}
C(t) & =E(X(t+1) \mid X(1)=1)-E(X(t+1) \mid X(1)=0) \\
& =E(P(X(t+1)=1 \mid X(1)=1)-E(P(X(t+1)=1) \mid X(1)=0) \\
& =\frac{D_{\infty}(t)\left(E\left(Z_{\infty}(t) \mid X(1)=1\right)-E\left(Z_{\infty}(t) \mid X(1)=0\right)\right)}{D_{\infty}(t)+\theta}=\frac{1}{\theta+1}=C(1) .
\end{aligned}
$$

The result agrees with (44). We estimate the conditional variance of $Z_{\infty}(t)$ for $X(1)=x$ as

$V\left(Z_{\infty}(t) \mid X(1)=x\right)=B^{2}\left(1+\frac{\theta}{t}\right)^{2} \cdot \frac{t-1}{(t+\theta)(1+\theta)} \simeq \frac{B^{2}}{1+\theta}=\frac{1}{\theta+1} \cdot \frac{(\alpha+x)(\beta+(1-x)}{(\theta+1)^{2}}$

Apart from the denominator of the prefactor, it agrees with the exact result of (5). 